\title{
Modification on surface oxide layer structure and surface morphology of niobium by gas cluster ion beam treatments
}

\author{
A. T. Wu, ${ }^{1, *}$ D. R. Swenson, ${ }^{2}$ and Z. Insepov ${ }^{3}$ \\ ${ }^{1}$ Thomas Jefferson National Accelerator Facility, 12000 Jefferson Avenue, Newport News, Virginia 23606, USA \\ ${ }^{2}$ Passport Systems Inc., 70 Treble Cove Road, Billerica, Massachusetts 01862, USA \\ ${ }^{3}$ Argonne National Laboratory, 9700 South Cass Avenue, Argonne, Illinois 60439, USA
}

(Received 20 November 2009; published 24 September 2010)

\begin{abstract}
Recently, it was demonstrated that significant reductions in field emission on $\mathrm{Nb}$ surfaces could be achieved by means of a new surface treatment technique called gas cluster ion beam (GCIB). Further study as shown in this paper revealed that GCIB treatments could modify surface irregularities and remove surface asperities leading to a smoother surface finish as demonstrated through measurements using a 3D profilometer, an atomic force microscope, and a scanning electron microscope. These experimental observations were supported by computer simulation via atomistic molecular dynamics and a phenomenological surface dynamics. Measurements employing a secondary ion mass spectrometry found that GCIB could also alter $\mathrm{Nb}$ surface oxide layer structure. Possible implications of the experimental results on the performance of $\mathrm{Nb}$ superconducting radio frequency cavities treated by GCIB will be discussed. First experimental results on $\mathrm{Nb}$ single cell superconducting radio frequency cavities treated by GCIB will be reported.
\end{abstract}

DOI: 10.1103/PhysRevSTAB.13.093504

PACS numbers: $29.20 .-\mathrm{c}, 29.90 .+\mathrm{r}, 34.35 .+\mathrm{a}, 81.05 .-\mathrm{t}$

\section{INTRODUCTION}

Recent experiments [1-4] have shown that the gas cluster ion beam (GCIB) technique is a highly desirable tool for treating $\mathrm{Nb}$ surfaces to reduce field emission. In order to use this new technique in an optimized fashion, it is important to understand what kind of effects this new tool has on the treated $\mathrm{Nb}$ surfaces.

In this paper, we report on the results of the measurements on the surfaces of $\mathrm{Nb}$ samples treated by various GCIB gas species employing a 3D profilometer, an atomic force microscope (AFM), a scanning electron microscope (SEM), and a dynamic secondary ion mass spectrometry (SIMS). Computer simulation via atomistic molecular dynamics and a phenomenological surface dynamics was employed to understand the experimental observations. It will be demonstrated in the following that GCIB treatments can not only modify surface morphology of $\mathrm{Nb}$ but also change its surface oxide layer structure. By adjusting GCIB treatment parameters and agent, it is possible to reduce field emission significantly and improve superconducting properties on the $\mathrm{Nb}$ surface, leading to a better performance for $\mathrm{Nb}$ based superconducting radio frequency (SRF) cavities.

\section{SAMPLES AND EXPERIMENTAL INSTRUMENTS}

The samples used here were the same samples as those for the study reported in Ref. [2]. The samples were

\footnotetext{
*andywu@jlab.org
}

fabricated from the same $\mathrm{Nb}$ batch. These were special samples designed particularly for doing field emission scans using the scanning field emission microscope built at JLab. After the fabrication, the samples were treated by the standard buffered chemical polishing (BCP) to remove a thickness of $150 \mu \mathrm{m}$ from the surfaces. After the chemical treatments, the samples were first rinsed with deionized (DI) water followed by ultrasonic cleaning with MICRO90 (a detergent manufactured by International Products Corporation) for one hour and then rinsed again with DI water. Finally, water on sample surfaces was blown away by a dry nitrogen gun.

The study was done using the surface instruments built in the surface science lab at JLab. For a detailed description of the instruments, please consult Ref. [5].

\section{MODIFICATION ON SURFACE MORPHOLOGY OF NB BY GCIB}

One of the most important effects from GCIB treatments is the ability to modify the morphology of the surface under treatments. This effect is relevant to the performance of $\mathrm{Nb}$ SRF cavities, since the smoother inner surface of a $\mathrm{Nb}$ SRF cavity tends to give better performance [6]. It is also one of the important factors contributing to the suppression of field emission as discussed in Ref. [2]. This section will deal with how GCIB treatments can modify the morphology of $\mathrm{Nb}$ surfaces. To study this effect, an atomic force microscope (AFM) and a high precision 3D profilometer are employed experimentally and computer simulation via atomistic molecular dynamics and a phenomenological surface dynamics is used theoretically. 
The ability of GCIB treatments for modifying Nb surfaces under the treatments manifests itself via the measurements of etching rates. The etching rates of $\mathrm{Nb}$ by $\mathrm{NF}_{3}+\mathrm{O}_{2}$, Ar, and $\mathrm{O}_{2}$ have been measured quantitatively [7]. $\mathrm{NF}_{3}+\mathrm{O}_{2}$ was found to have the highest etching rate of $5 \mathrm{~nm}^{*} \mathrm{~cm}^{2} / \mathrm{s}$ at $35 \mathrm{kV}$ acceleration voltage.

Typical examples of profilometer measurements on a $\mathrm{NF}_{3}+\mathrm{O}_{2}$ treated $\mathrm{Nb}$ sample are shown in Fig. 1 for the untreated and treated halves, respectively. In general, $\mathrm{NF}_{3}+\mathrm{O}_{2}$ GCIB treatment at $35 \mathrm{kV}$ makes the surface rougher. Typically the $\mathrm{rms}$ of the treated region is $615 \mathrm{~nm}$ over an area of $200 \times 200 \mu \mathrm{m}^{2}$ as compared with $315 \mathrm{~nm}$ for the untreated region. It seems that there are some shallow craters generated by $\mathrm{NF}_{3}+\mathrm{O}_{2}$ GCIB treatment on the treated region. Part of the reason for creating the craters can be due to the larger mass involved in $\mathrm{NF}_{3}$ clusters. Therefore mechanical impact on the treated surface is much larger than that when employing much lighter clusters such as, for instance, $\mathrm{O}_{2}$. More study is needed in order to optimize $\mathrm{NF}_{3}+\mathrm{O}_{2}$ GCIB treatments on $\mathrm{Nb}$.

Profilometer measurements on an $\mathrm{O}_{2}$ treated sample, on the other hand, did not show any clear differences between the treated and untreated regions as shown typically in Fig. 2. The rms extracted from the scans varies from location to location and it oscillated around $1.27 \mu \mathrm{m}$ depending on where the scans were done. The average rms did not correlate with a region regarding whether it was treated by $\mathrm{O}_{2}$ GCIB. Note here that although our samples come from the same batch the average roughness of this set of samples is different from that of the samples
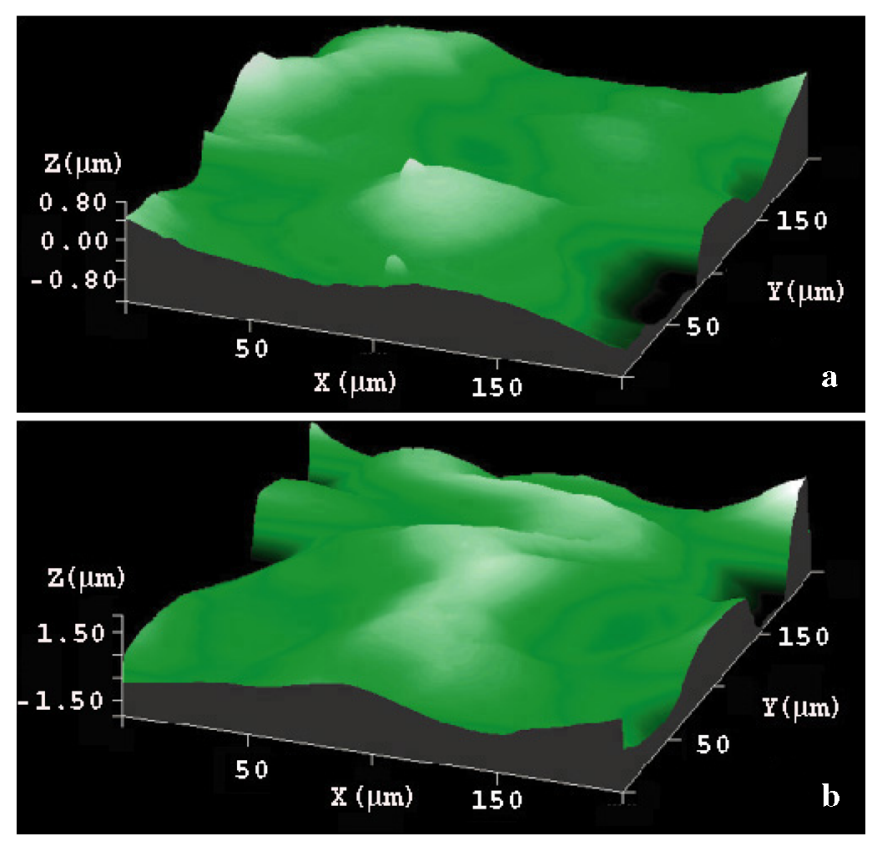

FIG. 1. Typical profilometer images of $200 \times 200 \mu \mathrm{m}^{2}$ of (a) an untreated region and (b) a treated region obtained on a $\mathrm{BCP} \mathrm{Nb}$ coupon treated by $\mathrm{NF}_{3}+\mathrm{O}_{2} \mathrm{GCIB}$.
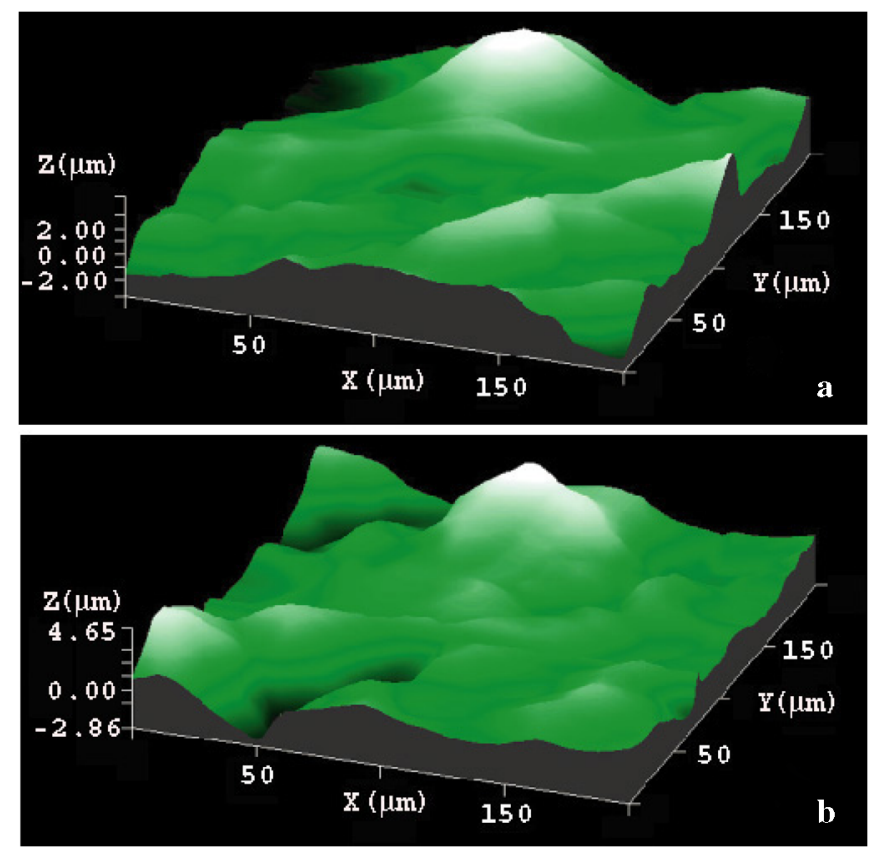

FIG. 2. Typical profilometer images of $200 \times 200 \mu \mathrm{m}^{2}$ of (a) an untreated region and (b) a treated region obtained on a $\mathrm{BCP} \mathrm{Nb}$ coupon treated by $\mathrm{O}_{2}$ GCIB.

for the $\mathrm{NF}_{3}+\mathrm{O}_{2}$ treatment study, indicating the possibility of the surface smoothness variation of $\mathrm{Nb}$ from one sheet to another even if they are obtained from the same batch.

However, we know that $\mathrm{O}_{2}$ GCIB treatments do etch away materials from the $\mathrm{Nb}$ surface [7]. Therefore we tried to do a more detailed study employing an AFM. In this case, a $\mathrm{Nb}$ coupon was divided into four quadrants as shown in Fig. 3. The region marked " $U$ " means that it was untreated, "P1" means it was treated at $25 \mathrm{kV}$, "P2" means it was treated at $5 \mathrm{kV}$, and "P1 + P2" means it was treated at $25 \mathrm{kV}$ first followed by treatment at $5 \mathrm{kV}$. This was inspired by the fact that GCIB treatments with an initial etch rate followed by one or more lower etch rates

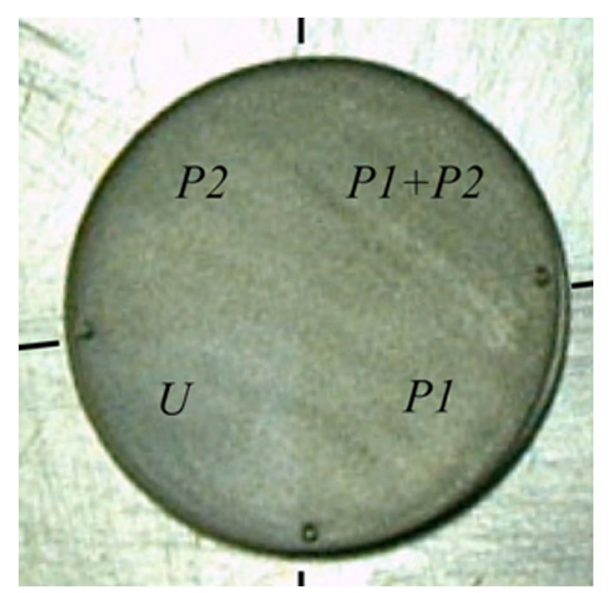

FIG. 3. A Nb coupon was masked into equal quadrants for treatment with high and low energies $\mathrm{O}_{2}$ GCIB (see the text for more details). 

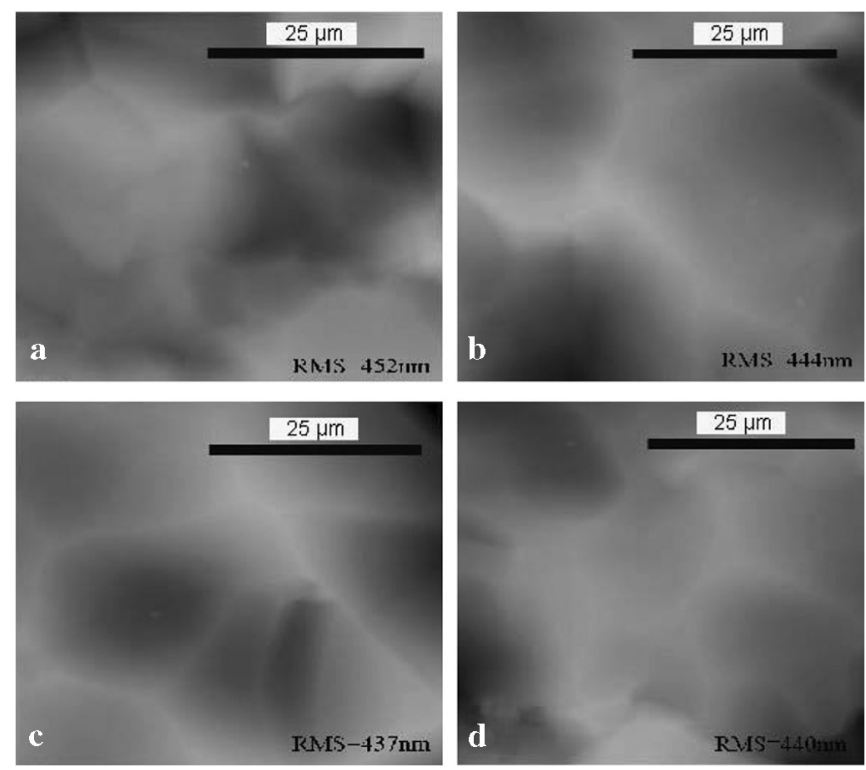

FIG. 4. Typical AFM images of $50 \times 50 \mu \mathrm{m}^{2}$ obtained on the sample shown in Fig. 3: (a) for the untreated region, (b) for the $\mathrm{P} 1$ treated region, (c) for the P1 + P2 treated region, and (d) for the $\mathrm{P} 2$ treated region.

can minimize the remaining roughness of the final surface and minimize material removal in order to attain a desirable level of smoothness [8]. AFM measurements were carried out using a Nanoscope IV controller dimension $310^{\mathrm{TM}}$ SPM head (model name). Tapping mode was used in all the observations shown in this section.

Figure 4 shows typical AFM images obtained on all four quadrants of the sample with a scanning size of $50 \mu \mathrm{m} \times$ $50 \mu \mathrm{m}$. The untreated region is rougher than the rest of the four quadrants. The $\mathrm{P} 1+\mathrm{P} 2$ treated region is indeed smoother than that treated by either P1 or P2, which is consistent with the suggestions made in Ref. [8]. It seems that the region treated at $5 \mathrm{kV}$ is a little smoother than that treated at $25 \mathrm{kV}$.

\section{COMPUTER SIMULATION}

To understand the intrinsic mechanism associated with the modifications of morphology on Nb surfaces by GCIB treatments, computer simulations through molecular dynamic modeling were employed. $\mathrm{Ar}$ and $\mathrm{O}_{2}$ were selected as the species for the GCIB clusters. The Nb surface that would be treated by GCIB was supposed to be $(1,0,0)$. Assuming that each cluster was multiply charged and contained 429 molecules or atoms, it was found that heavier GCIB species such as Ar could generate larger and deeper craters than those generated by lighter GCIB species on a $\mathrm{Nb}$ surface as shown in Fig. 5. In the simulation here, the kinetic energy of Ar was assumed to be $125 \mathrm{eV} /$ atom and that of $\mathrm{O}_{2}$ was $100 \mathrm{eV} /$ molecule. This could explain the results found from the profilometer measurements on the samples treated by $\mathrm{O}_{2}$ as shown in Figs. 1 and 4.
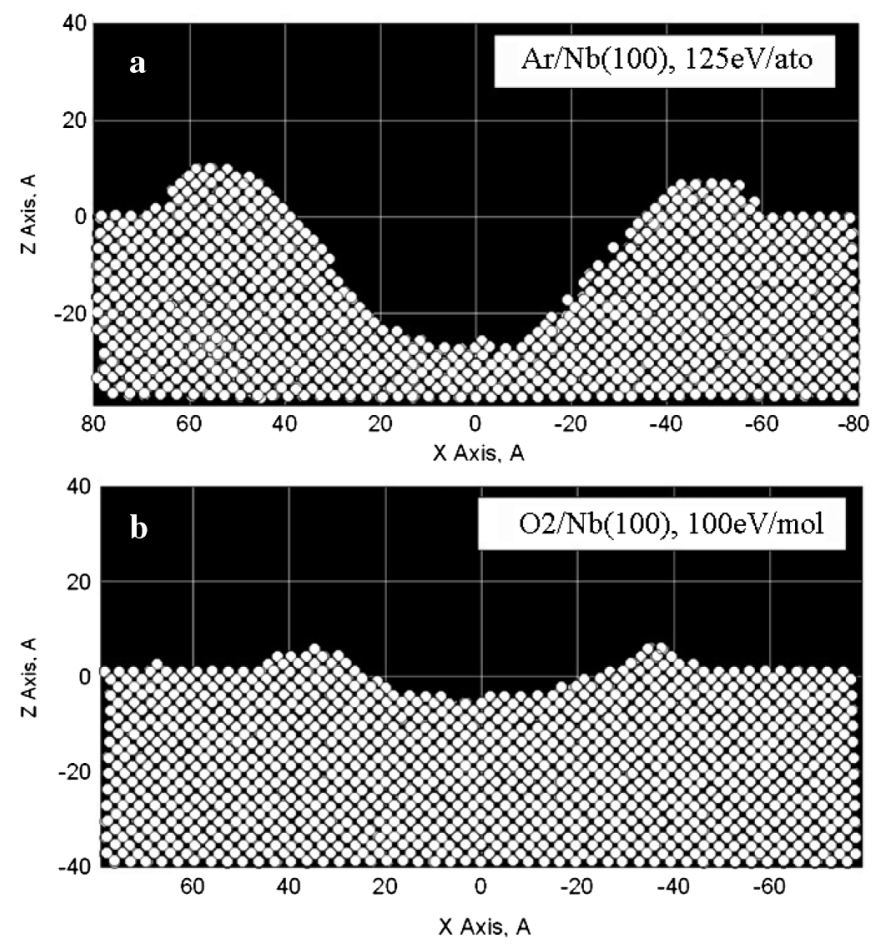

FIG. 5. Craters formed on the surface of (100) $\mathrm{Nb}$ treated with (a) clusters of $429 \mathrm{Ar}$ at $125 \mathrm{eV} /$ atom, and (b) clusters of $429 \mathrm{O}_{2}$ at $100 \mathrm{eV} /$ molecule, as calculated by computer simulation via molecular dynamics.

A smoothing effect by GCIB treatments was demonstrated by modeling a $\mathrm{Nb}$ surface containing two types of surface tips with significantly different sizes. One tip was a narrow and tall hill with a typical diameter of a few nm. The other was a wide and short hill having a typical diameter of many tens of $\mathrm{nm}$. Both tips had equal volumes and were schematically shown in Fig. 6(a). The total modeled area was in the order of $10^{6}-10^{7} \AA^{2}$, and this area was irradiated by up to $100030 \mathrm{keV} \mathrm{O}_{2}$ clusters. The clusters randomly bombarded the whole area of the simulation cell. The cluster dose was in the order of $10^{3}-10^{4}$ cluster/cell. The typical irradiation parameters used for surface smoothing were as follows: cluster ion doses were in the range of $10^{12}-10^{15} \mathrm{ion} / \mathrm{cm}^{2}$, average cluster sizes were in the order of $10^{3}$ atoms or molecules, and the total cluster energies was $30 \mathrm{keV}$. Displacements of surface particles after the cluster impact were modeled in accordance with the probability, obtained in our molecule dynamic simulation of a single cluster ion impact on a flat or inclined $\mathrm{Nb}$ surface.

Figure 6 demonstrates the results of our mesoscale simulations for $\mathrm{Nb}$ surface smoothening. The residual roughness is always defined by the geometry of an individual crater and increases with the increase of the total cluster ion energy. This explains why the region treated at $25 \mathrm{kV}$ in Fig. 4 is a bit rougher than that treated at $5 \mathrm{kV}$. The simulation showed that the narrower hill could be removed 

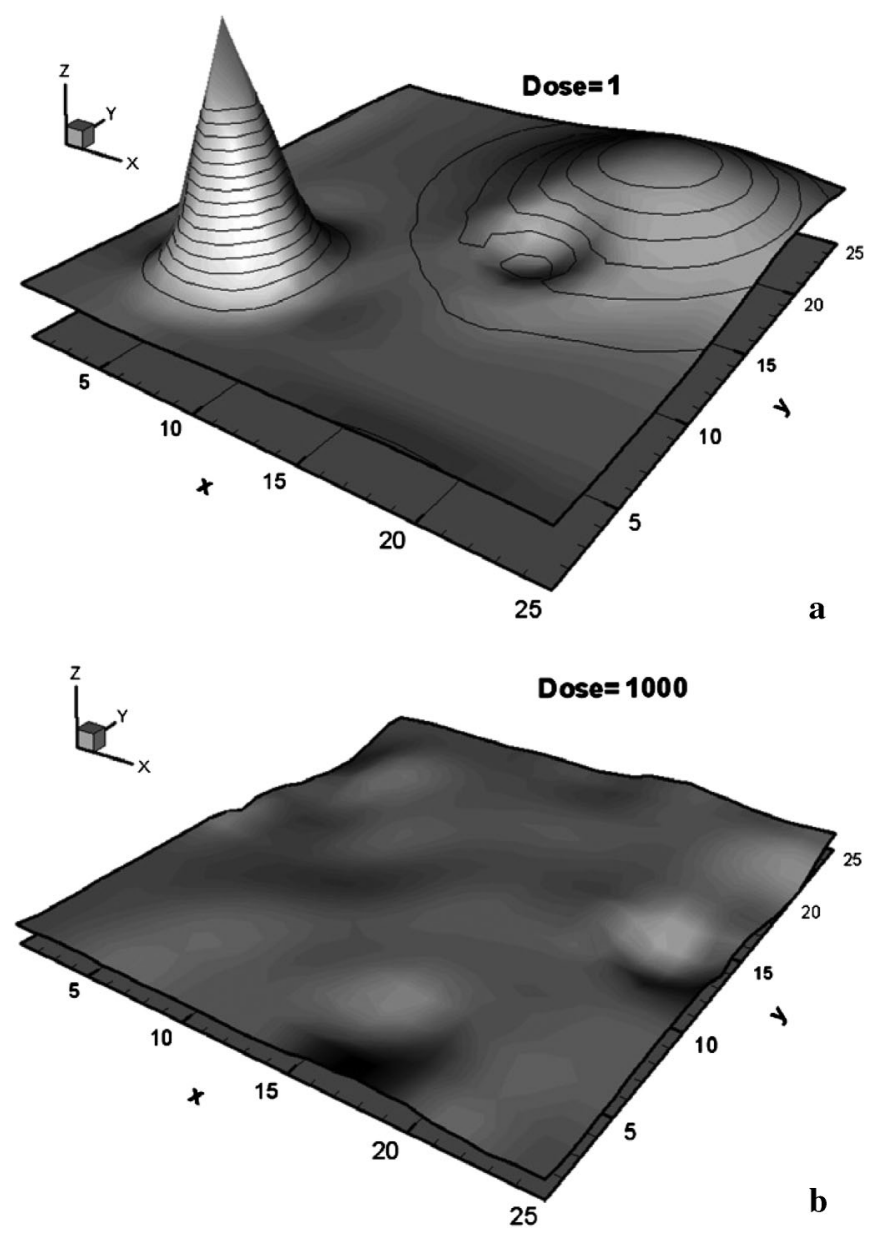

FIG. 6. Results of mesoscale modeling of a Nb surface irradiated by an $\mathrm{O}_{2}$ cluster ion beam at a dose of $10^{13}$ ions $/ \mathrm{cm}^{2}$. The cluster energy was $30 \mathrm{keV}$ and the cluster size was about 3000 oxygen molecules in a cluster. The surface contained two types of features: narrow and tall and wide and short [represented in (a)].

by an irradiation dose that was 5 times lower than that required for the blunt hill. The larger the surface bump is in the horizontal plane, the higher irradiation dose is needed to completely remove the hill and smooth the surface. It is known that the narrower hills have a higher chemical potential than those with a larger diameter. Therefore chemically inactive GCIB surface treatments should remove the narrow hills faster than the wider ones. Computer simulation seems to suggest that the surface smoothing of $\mathrm{Nb}$ is mostly done by physical removal of the hills through mechanical interactions between the incoming GCIB clusters and the atoms of the treated surfaces rather than by chemical reaction. For details about this computer simulation study, please read Ref. [9].

\section{MODIFICATIONS OF NB SURFACE OXIDE LAYER STRUCTURE BY GCIB}

It is well known that the performance of Nb SRF cavities depends critically on their surface top layer of about $50 \mathrm{~nm}$ deep. The outmost layer of any $\mathrm{Nb}$ surface is always covered with an oxide layer. We used to believe that the thickness of the oxide layer was approximately $6 \mathrm{~nm}$. However, the latest atomically resolved TEM cross-section images [10] show that the oxide layer is much thinner for $\mathrm{BCP}$ and buffered electropolishing [11] treated $\mathrm{Nb}$ samples. Most of the oxides in this top layer are $\mathrm{Nb}$ pentoxides that are dielectric and are generally believed to have no negative effects on the performance of Nb SRF cavities. However, some $\mathrm{Nb}$ suboxides might exist [10] at the interface between the $\mathrm{Nb}_{2} \mathrm{O}_{5}$ and pure $\mathrm{Nb}$ such as, for instance, $\mathrm{Nb}_{2} \mathrm{O}$ or $\mathrm{NbO}$ or others that may not be superconducting or may be superconducting at lower critical temperatures than that of pure $\mathrm{Nb}$. These suboxides can definitely cause rf losses and degrade the rf performance of $\mathrm{Nb}$ cavities. It is shown in this section that GCIB treatments can modify the surface oxide layer structure of $\mathrm{Nb}$.

To study the modification of the surface oxide layer structure of $\mathrm{Nb}$ by GCIB treatments, a home-made dynamic SIMS system [5] was employed. $\mathrm{Ar}^{+}$was used as the primary ion source. Measurements were done at a vertical incident angle, $2.5 \mathrm{keV}$, and $85 \mu \mathrm{A} / \mathrm{cm}^{2}$. Both whole spectrum and depth profile were recorded. Depth profile measurements were done via a method described in Ref. [12]. Nb coupons were treated by $\mathrm{NF}_{3}+\mathrm{O}_{2}, \mathrm{O}_{2}$, and $\mathrm{N}_{2}$. Ar was not used due to the fact that it might create confusion for the interpretation of the experimental results since the primary ion source was $\mathrm{Ar}^{+}$.

Figure 7 shows the whole spectra for a $\mathrm{Nb}$ coupon of which half was treated by GCIB $\mathrm{O}_{2}$ and the other half was untreated. Depth profile measurements are shown in Fig. 8. From Figs. 7 and 8, we can see the following: (1) The $\mathrm{Nb}$
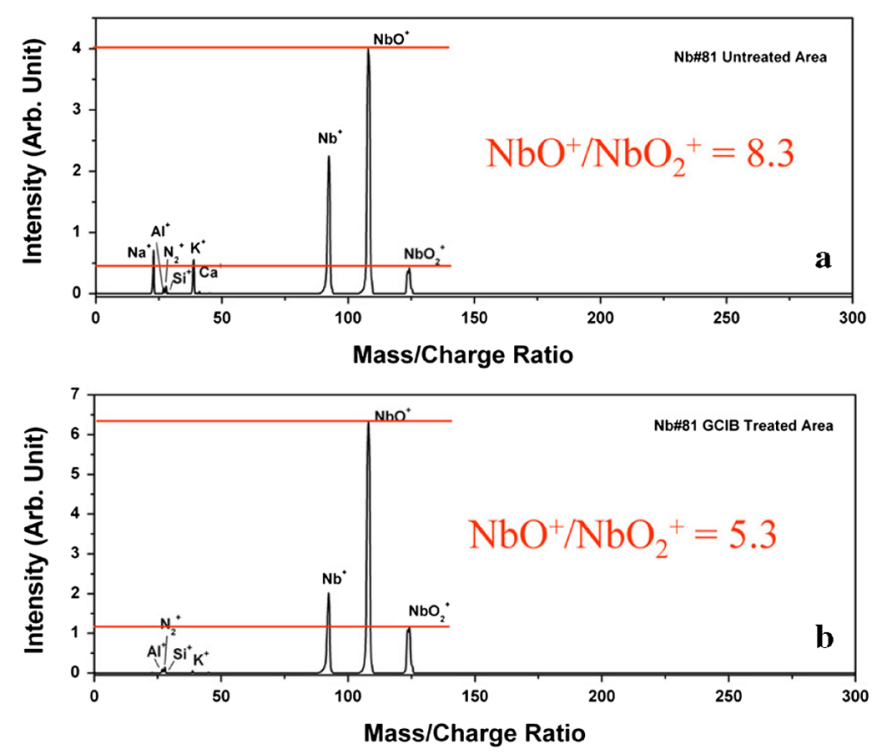

FIG. 7. Typical SIMS whole spectrum measurements done on (a) an untreated region of a BCP Nb coupon treated by $\mathrm{O}_{2}$ GCIB and (b) a treated region of a $\mathrm{BCP} \mathrm{Nb}$ coupon treated by $\mathrm{O}_{2}$ GCIB. 


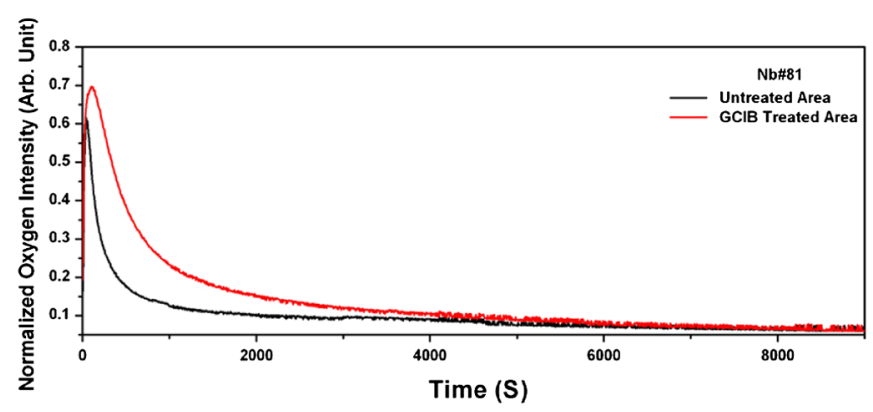

FIG. 8. Typical SIMS depth profile measurements done on a $\mathrm{BCP} \mathrm{Nb}$ coupon treated by $\mathrm{O}_{2}$ GCIB.

surface is cleaner after the GCIB treatment. Elements such as $\mathrm{Na}$ and $\mathrm{Ca}$ disappear completely after the treatments, while the intensities of other peaks (apart from $\mathrm{Nb}$ and its oxides) reduce. (2) A significant amount of oxygen is introduced to the surface layer of $\mathrm{Nb}$ and the thickness of the oxide layer of the treated area is increased as compared with that of the untreated area. The increase in the thickness of the top oxide layer contributes to the suppression of field emission as discussed in Ref. [2]. This is because after $\mathrm{O}_{2}$ GCIB treatment the particulates are attached to a $\mathrm{Nb}$ surface that has a dielectric layer with a thickness more than double than that before the $\mathrm{O}_{2}$ GCIB treatment, which makes the onset field of an emitter higher in order to sustain field emission. The mechanism regarding how $\mathrm{O}_{2}$ GCIB treatments could increase the thickness of the oxide layer is not completely clear at the present moment, since implantation is expected to be minimal in GCIB treatments as discussed in the previous sections. However, somehow probably $\mathrm{O}_{2}$ GCIB treatments can enhance oxygen diffusion into the interior of $\mathrm{Nb}$. (3) The cracking patterns of $\mathrm{Nb}$ and its oxides change significantly after the treatment. For instance, from the two whole spectra we see that $\mathrm{Nb} / \mathrm{NbO} / \mathrm{NbO}_{2}$ is $6 / 11 / 1$ for the untreated area and $6 / 22 / 4$ for the treated area. (4) The normalized maximum intensity of the oxygen content is 0.084 higher for the treated area. This is an increase of $13.7 \%$ than that of the untreated area. This implies that on the treated area, there can be an oxide layer with an oxidation state of $\mathrm{Nb}_{2} \mathrm{O}_{5}+x$ $(x>0.5)$. It is highly plausible that the extra oxygen atoms exist as interstitial atoms in the amorphous $\mathrm{Nb}_{2} \mathrm{O}_{5}$ layer. It seems that the treatment is not optimized, since the penetration of oxygen into the $\mathrm{Nb}$ surface is much too deep.

To explore the oxygen penetration effect, a $\mathrm{Nb}$ coupon was treated with different energies and durations in a way identical to that shown in Fig. 3. The treatment duration for the $\mathrm{P} 1+\mathrm{P} 2$ region was twice as much as that for the $\mathrm{P} 1$ or the $\mathrm{P} 2$ region. Oxygen depth profile data are plotted in Fig. 9. Figure 9 tells us that the depth of oxygen penetration depends only on the duration of the GCIB treatment and has nothing to do with the treatment energy inside the energy window selected in this study. Higher treatment energy increases only the maximum intensity of the
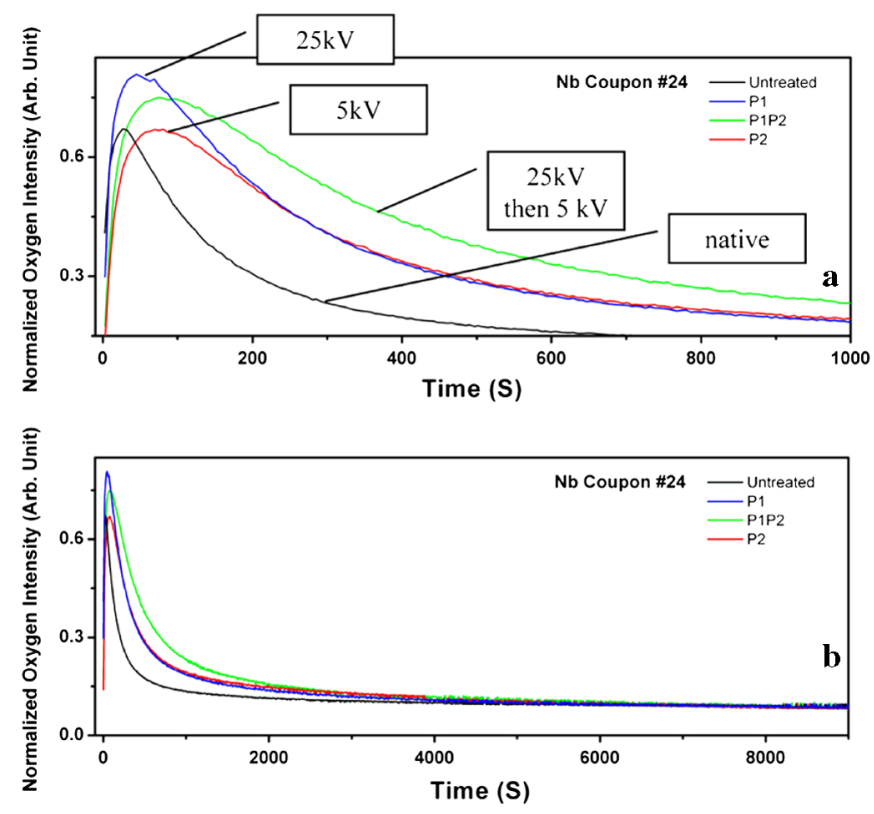

FIG. 9. Typical SIMS depth profile measurements done on the BCP Nb coupon shown in Fig. 3. Part (a) shows close-up plots of the depth profile data. Part (b) shows the depth profiles measured on every quadrant.

oxygen peak and its peak location, implying that probably more interstitial oxygen atoms exist in the $\mathrm{Nb}_{2} \mathrm{O}_{5}$ layer for the region treated at $25 \mathrm{kV}$. Therefore, GCIB treatment time has to be optimized in order to create a sharp interface between $\mathrm{Nb}_{2} \mathrm{O}_{5}$ and pure $\mathrm{Nb}$. This work has not been done yet.

SIMS measurements were also done on $\mathrm{NF}_{3}+\mathrm{O}_{2}$ and $\mathrm{N}_{2}$ treated $\mathrm{Nb}$ coupons. Because of the limited space here, readers are referred to Ref. [1] for the details. In all cases studied up to now, modifications of surface oxide layer structure were found.

\section{FIRST RF MEASUREMENTS ON NB SRF CAVITIES TREATED BY GCIB}

It has been illustrated experimentally in the previous sections that GCIB treatments could be hoped to have some beneficial effects on the performance of $\mathrm{Nb}$ based SRF cavities in some indirect ways through surface measurements and computer simulation. It would be nice to see how rf performance of a $\mathrm{Nb}$ SRF single cell cavity is affected by GCIB treatments in reality. In this section, we report our initial attempts in this direction.

An experimental setup [1] was constructed at Epion to allow GCIB treatments on $\mathrm{Nb}$ single cell cavities. A $1.3 \mathrm{GHz} \mathrm{Nb}$ single cell cavity was made at JLab and cleaned and pumped down to $10^{-7}$ Torr and shipped to Epion under vacuum. Then staff at Epion opened the cavity shipping container in a class 10 environment and transferred the cavity into the GCIB cavity treatment chamber [1]. Care was taken not to introduce any particulates into 


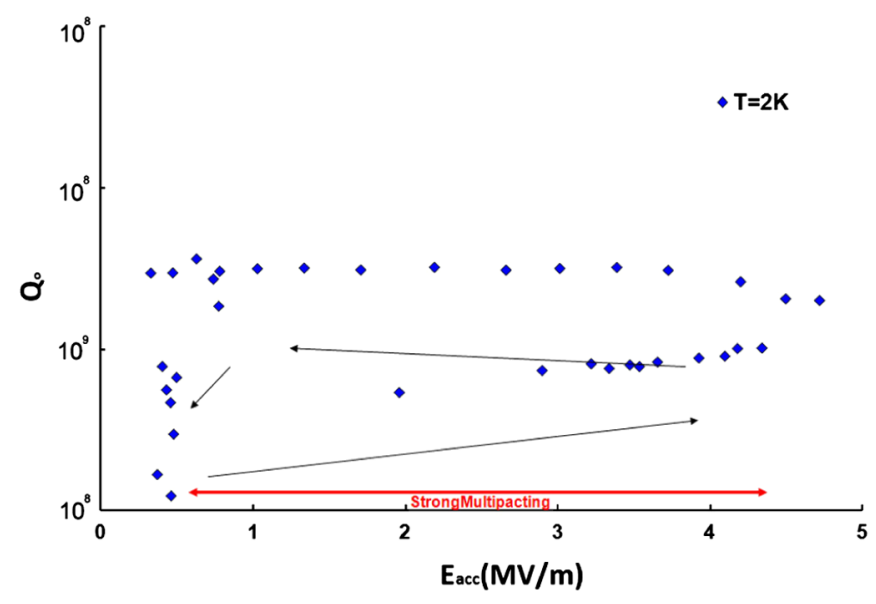

FIG. 10. Excitation curve measured at $2 \mathrm{~K}$ for the $\mathrm{O}_{2}$ GCIB treated $1.3 \mathrm{GHz} \mathrm{Nb}$ SRF single cell cavity right after the treatment.

the cavity before $\mathrm{O}_{2}$ GCIB treatment. After the treatment, the process was reversed and the cavity was rf tested at JLab.

Figure 10 shows the first rf test result on the $\mathrm{O}_{2} \mathrm{GCIB}$ treated $\mathrm{Nb}$ cavity right after receiving the cavity from Epion. Strong multipacting was found due to contamination on the treated surface as a result of bad handling during the transportation of the cavity between JLab and Epion [1]. To remove the contamination of the first $\mathrm{O}_{2}$ GCIB treated cavity, high pressure water rinse (HPWR) was employed. Figure 11 shows the result of the rf test after the HPWR. The test was limited by quenching at $22.7 \mathrm{MV} / \mathrm{m}$. Field emission was not a problem anymore after the HPWR, implying that the contamination was not intrinsic to the $\mathrm{O}_{2}$ GCIB treatment and the field emission could be avoided if a better cavity handling procedure was established. Although the first measured excitation curve for the cavity was not that impressive, $Q_{0}$ measured at

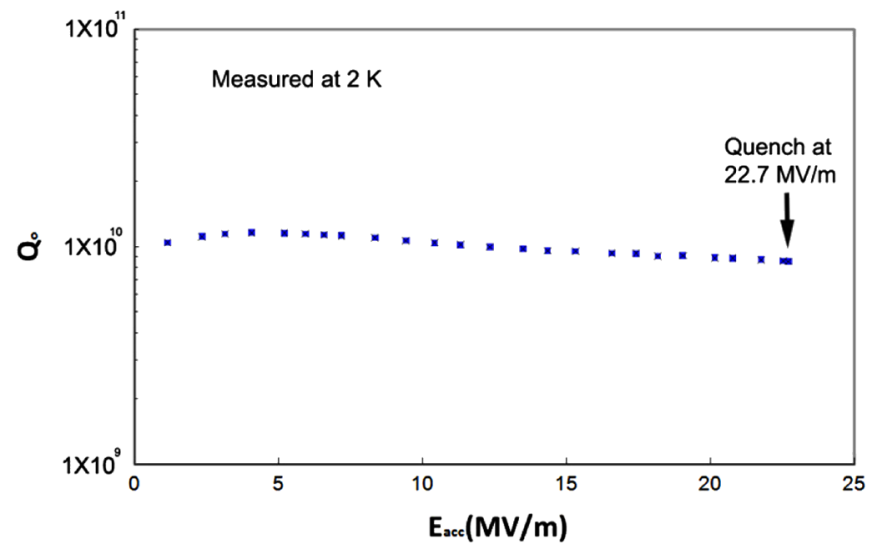

FIG. 11. Excitation curve measured at $2 \mathrm{~K}$ for the $\mathrm{O}_{2}$ GCIB treated $1.3 \mathrm{GHz} \mathrm{Nb} \mathrm{SRF}$ single cell cavity after high pressure water rinse.
$4.5 \mathrm{~K}$ was $7.5 \times 10^{8}$ that was even a little better than that after the low temperature baking at $120^{\circ} \mathrm{C}$ for 48 hours-a procedure commonly used for removing the high-field $Q$ slope $[13,14]$. Furthermore, the superconducting gap value extracted from the $Q_{o}$ vs $1 / T$ measurement (see Fig. 12) for the $\mathrm{O}_{2}$ GCIB treatment is $\Delta / \mathrm{kT}_{c}=2.04 \pm 0.01$ which is larger than 1.85 commonly found on Nb SRF cavities after the low temperature baking. In the extraction process, a computer code [15] based on the full BCS formalism was employed for both cases before and after the GCIB treatment. A similar result was also found on a $3.9 \mathrm{GHz}$ cavity that was first treated at $35 \mathrm{kV}$ by $\mathrm{O}_{2} \mathrm{GCIB}$ followed by a $\mathrm{O}_{2}$ GCIB treatment at $5 \mathrm{kV}$. Therefore GCIB treatments can enhance the superconducting gap value of $\mathrm{Nb}$ by assuming that the superconducting transition temperature of the bulk is not altered by this GCIB surface treatment. It is important to point out here that the $T_{c}$ during the extraction process is fixed at $92.5 \mathrm{~K}$ for both GCIB treated and baked cases. Our cavity data seem to indicate that the effect of $\mathrm{O}_{2}$ GCIB treatments to the cavity performance is similar to that after the low temperature baking at $120^{\circ} \mathrm{C}$ for 48 hours. It was concluded in Ref. [1] that the enhanced superconducting gap structure in the GCIB treated $\mathrm{Nb}$ single cell cavities is a result of oxygen diffusion into the pure $\mathrm{Nb}$ lattice right underneath the surface oxide layer on the $\mathrm{Nb}$ surfaces as discussed in Sec. V. If this diffusion process increases the thickness of the surface pentoxide layer while creating a sharper interface between the pentoxide layer and the pure $\mathrm{Nb}$ bulk, we would anticipate an improved superconducting property on the surface. Clearly, the pioneer work described in this section shows that the superconducting properties (and thus the performance of $\mathrm{Nb}$ SRF cavities) of a $\mathrm{Nb}$ surface can be altered through $\mathrm{O}_{2}$ GCIB treatments. Optimization of the parameters of $\mathrm{O}_{2}$ GCIB treatments can lead to improved rf performance for $\mathrm{Nb}$ SRF cavities. The idea of using the GCIB technique for treating a multicell Nb SRF cavity is outlined in Ref. [1].

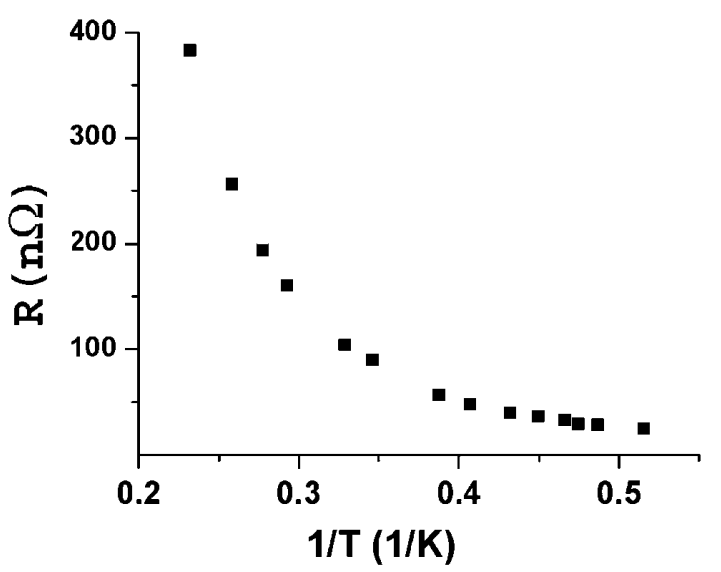

FIG. 12. Residual resistance measured as a function of $1 / T$ on the $\mathrm{O}_{2}$ GCIB treated $1.3 \mathrm{GHz} \mathrm{Nb}$ SRF single cell cavity. 


\section{SUMMARY}

To summarize, this paper reported on the investigation of the change of $\mathrm{Nb}$ surface morphology and oxide layer structure by GCIB treated via measurements by a 3D profilometer, an AFM, an SEM, and a dynamic SIMS system. Theoretically computer simulation through atomistic molecular dynamics and a phenomenological surface dynamics was employed to help understand the experimental results. It was found that GCIB treatments could remove sharp features on $\mathrm{Nb}$ surfaces and could sometimes smash particulates into some smaller and smoother pieces that might not field emit. A SIMS study showed that surface oxide layer structure could be tailored with GCIB treatments by using an appropriate treating agent such as, for instance, $\mathrm{O}_{2}$. Because of its effectiveness at changing the depth and composition of the surface oxide layer structure of $\mathrm{Nb}$, GCIB might be a key to understanding and overcoming the limitations of the high-field $Q$ slope. First $\mathrm{rf}$ test results on Nb SRF single cell cavities show that $\mathrm{O}_{2}$ GCIB treatments can enhance the $\mathrm{Nb}$ superconducting gap value leading to an improved rf performance of the cavities. A more detailed description of this work will appear in a chapter of a book published by NOVA Science Publishers [1]. More work is needed in order to fully explore this research topic.

\section{ACKNOWLEDGMENTS}

A. T. Wu would like to thank Peter Kneisel for his support in rf tests of the cavity and G. Ciovati for some useful discussions and confirmation regarding the fitting to Fig. 12. This work was supported by the U.S. Department of Energy, Contract No. DE-AC05-84-ER40150.

[1] A. T. Wu et al., in Neural Computation and Particle Accelerators: Research, Technology and Applications (NOVA Science Publishers, New York, 2009).

[2] A. T. Wu and D. R. Swenson, in Proceedings of the 14th SRF Conference, Germany, 2009, THPPO065.

[3] D. R. Swenson, A. T. Wu, E. Degenkolb, and Z. Insepov, Nucl. Instrum. Methods Phys. Res., Sect. B 261, 630 (2007).

[4] Z. Insepov, J. Norem, A. Hassanein, and A. T. Wu, AIP Conf. Proc. 1099, 46 (2009).

[5] A.T. Wu, in Proceedings of the 11th SRF Workshop, Germany, 2003, ThP13.

[6] B. Visentin, in Ref. [5], TU001.

[7] D. R. Swenson, A. T. Wu, E. Degenkolb, and Z. Insepov, AIP Conf. Proc. 877, 370 (2006).

[8] http://www.freepatentsonline.com/6805807.html.

[9] Z. Insepov et al., Vacuum 82, 872 (2008).

[10] A. T. Wu et al., in Ref. [2], TUPPO041.

[11] A. T. Wu et al., Appl. Surf. Sci. 253, 3041 (2007).

[12] A. T. Wu, Physica (Amsterdam) 441C, 79 (2006).

[13] P. Kneisel et al., in Proceedings of the 8th SRF Workshop, Abano, Terme, 1997, p. 463.

[14] E. Kako et al., in Ref. [13], 491.

[15] G. Ciovati, Ph.D. thesis, Old Dominion University, Norfolk, Virginia, 2005. 